\title{
An Early Warning Model of Physical Exercise Training Based on Heart Rate and Time
}

\author{
Jun $\mathrm{SU}^{1,2}$, Song ZHANG ${ }^{1}$, Lin YANG ${ }^{1,{ }^{*}}$ and Xiao-he $\mathrm{LI}^{3}$ \\ ${ }^{1}$ College of Life Science and Bioengineering, Beijing University of Technology, Beijing, \\ 100124, China \\ ${ }^{2}$ Youth Sports Department, Beijing Municipal Bureau of Sports, Beijing, 100075, China \\ ${ }^{3}$ ZhongShiHongLi (Beijing) Health Technology Co. Ltd \\ ${ }^{*}$ Corresponding author
}

Keywords: Exercise heart rate, Early warning system, Fitness and health monitoring.

\begin{abstract}
In recent years, with a gradual decline of teenagers' fitness and health monitoring data in our country, physical health of teenagers is becoming a more and more important problem. The traditional evaluation method can test teenagers' health condition by spot checks every year. However it is difficult to analyze the changes of the teenagers' physical data. In the present paper, an early warning system of physical exercise based on exercise heart rate and time is proposed. The system classifies the intensity of movement by exercise heart rate and time, and then calculates the load and the score according to the evaluation standards we put. To verify the maneuverability of the evaluation theory model, real-time monitoring of heart rate during physical education class in a high school is conducted. Results show that based on exercise heart rate and time, the score of teenagers' exercise condition can be obtained, which can reflect the daily exercise of each person intuitively.
\end{abstract}

\section{Introduction}

With the development of society and the improvement of people's income, the children's physical constitution in China has not been developed. On the contrary, in recent years, the students' physical monitoring data show a gradual downward trend, children's physical health is becoming increasingly prominent. The main reason for the decline of children's physical fitness in our country is the decline in the level of physical activity $[1,2]$. A survey shows that only $18.3 \%$ of middle school students spend more than one hour a day.

At present, students' physical health standard test is carried out by the school for supervision and appraisal, which is the conclusion of the monitoring. This monitoring method is used in the form of spot checks and standard, the monitoring data can promote the development of school sports work. However, during in the actual operation process, it is difficult to compare and analyze the data changes in the students' physical fitness test, and then reflect the scientific nature of the sports load of middle school in the whole year [3,4].

Process monitoring is the daily or weekly exercise or load monitoring with a small device worn by the students. This can determine the number of outdoor sports students, the duration of the exercise, as well as the intensity of exercise (only to achieve effective exercise intensity can play an enhanced physical purpose), and other indicators. 
Along with the development of the monitoring device of the wearable motion parameters based on the Internet of things, as well as the development of the sensor technology provide the technical feasibility of the process monitoring of the students' daily physical exercise. The smart bracelet, which has the core of the three axis acceleration sensor, can record the movement of people and calculate the energy consumption. In competitive sports, athletes wearing the GPS module can record the movement track, distance and speed. In order to collect the heart rate, the heart rate is a kind of process monitoring tool which is commonly used in sports or the general population.

\section{Method}

\section{Theoretical Analysis of the Establishment of Early Warning Model}

Current evaluation of sports intensity indicators including heart rate, oxygen uptake, subjective fatigue, MET, etc.. Only heart rate can be the index which can be simple, direct measuring, and large sampling at the same time. Although the heart rate has some limitations, such as differences between different people, heart rate has good relationship with exercise intensity and can be used to evaluate the intensity of exercise in the same individual, in certain period of time and in a certain period of exercise intensity [5-7].

Combined with domestic and international authoritative children's sports training standards, prescriptions and suggestions, from the angle of accuracy and feasibility, the ideal standard and the qualified standard of motion quality monitoring was established, as shown in Table 1. The whole motion intensity is divided into three levels: low intensity exercise, medium intensity exercise and high intensity exercise (including extra high intensity exercise) according to the motion of the heart rate collected. Then the movement time of each intensity exercise was calculated. The exercise was scored according to the established evaluation criteria. In this study, we evaluated the exercise load from two aspects: exercise intensity and duration.

Table 1. Exercise quality monitoring standard.

\begin{tabular}{|l|l|l|l|}
\hline Standard & Exercise intensity & Exercise duration & $\begin{array}{l}\text { Exercise times } \\
\text { per week }\end{array}$ \\
\hline Ideal Standard & $\begin{array}{l}\text { Medium intensity exercise: } \\
40-59 \% \text { heart rate reserve } \\
\text { High intensity exercise: } \\
60-84 \% \text { heart rate reserve }\end{array}$ & $\begin{array}{l}\text { Medium and high } \\
\text { intensity exercise of } \\
\text { each not less than 30 } \\
\text { minutes }\end{array}$ & 7 \\
\hline Qualified Standard & $\begin{array}{l}\text { Medium intensity exercise: } \\
40-59 \% \text { heart rate reserve } \\
\text { High intensity exercise: } \\
60-84 \% \text { heart rate reserve }\end{array}$ & $\begin{array}{l}\text { A total of not less } \\
\text { than 60 minutes of } \\
\text { medium and high } \\
\text { intensity exercise }\end{array}$ & 5 \\
\hline
\end{tabular}

\section{Monitoring Method of Early Warning Model}

The intensity of exercise is classified according to the heart rate reserve as shown in Table 2. Among them, the maximum heart rate $H_{\mathrm{max}}=220-$ age [8]. If the actual maximum heart rate in the exercise exceeds the calculated heart rate, the actual maximum heart rate was used. A maximum heart rate in a certain period (such as one week) was used. Quiet heart rate: the heart rate recorded in the quiet state. A quiet heart rate in a certain period (such as one week) was used. Heart rate reserve: HRR= quiet heart rate + (the maximum heart rate - quiet heart rate) $*$ heart rate reserve percentage. 
Table 2. Intensity classification method.

\begin{tabular}{|l|l|}
\hline Intensity exercise & Heart rate range \\
\hline Low & quiet heart rate-40\% heart rate reserve \\
\hline Medium & $40 \%$ heart rate reserve-60\% heart rate reserve \\
\hline High & $60 \%$ heart rate reserve-85\% heart rate reserve \\
\hline Extra high & More than $85 \%$ heart rate reserve \\
\hline
\end{tabular}

According to the above method, each student's sports intensity was classified. All physical activity is divided into low intensity, medium intensity, high intensity, extra high intensity according to the intensity. Their final time was calculated. In calculating the amount of motion, the high intensity and the extra high intensity were combined calculated to the high intensity. The extra high intensity was not a separate statistical index, but only was a warning of its sports risk. After a certain period of time of monitoring and calculating in each student's physical education and extra-curricular activities, the next step can be carried on.

\section{Evaluation Method of Early Warning Model}

According to the intensity of the oxygen uptake, the intensity of the exercise was quantitated: the low intensity of the $0-40 \%$ reserve aerobic capacity, medium intensity $40-60 \%$ and high intensity for $60-85 \%{ }^{[9]}$. The mean of low intensity, medium intensity and high intensity are $20 \%, 50 \%$ and $72.5 \%$, respectively, and the intensity coefficient is simplified to 1,2 and 3 respectively.

The time (min) of each intensity exercise is multiplied by the intensity factor, and the load of each intensity is obtained. For example: every day, the ideal exercise load for each the medium and high intensity of 30 minutes, the calculation results are: Load $=30 * 3+30 * 2=150$.

\section{Result}

The actual amount of daily exercise load was calculated. Then the ratio of the amount of daily exercise load and the ideal exercise load (150) was calculated and multiplied by 100 to get a daily score. Table 3 is a subject's result of one week's score of exercise.

Table 3. A subject's result of one week's score of exercise.

\begin{tabular}{|c|c|c|c|c|c|c|c|c|}
\hline & Monday & Tuesday & Wednesday & Thursday & Friday & $\begin{array}{c}\text { Amount for } \\
\text { week }\end{array}$ & $\begin{array}{c}\text { Mean } \\
\text { value }\end{array}$ & $\begin{array}{c}\text { Mean value } \\
\text { for 7 days }\end{array}$ \\
\hline $\begin{array}{c}\text { Exercise } \\
\text { load }\end{array}$ & 81 & 141 & 144 & 131 & 106 & 603 & 121 & 86 \\
\hline Score & 54 & 94 & 96 & 88 & 71 & 402 & 80 & 57 \\
\hline
\end{tabular}

It can be seen that the average score of the students in each exercise day was 80 , but only 5 times exercise a week, so the average score was 57 per day. The above is only for the exercise activity after school, not all day. Through the above method, a certain period of time of exercise load of each student can be quantified. The score can be clearly given the completion and degree of the ideal exercise load.

\section{Discussion}

Drawing on the concept of the process monitoring of competitive sports, an early warning model of physical training condition based on exercise intensity and exercise time was proposed, in the full understanding of students' physical condition, students' 
physical exercise status, monitoring evaluation mechanism and the characteristics of existing wearable devices.

Through the suggestion of the domestic and international sports experts, the monitoring and early warning model based on the movement of the heart rate and time was established. The students' score of the exercise load level was got as the basis which can be used as a reference value for students' physical training effect or early warning.

\section{Acknowledgments}

This research was financially supported by Projects of Beijing Municipal Commission of Education (PXM2015_014204_500064, PXM2016_014204_500079), Project of Excellent talent of Organization Department of Beijing Municipal Party Committee (2014000020124G044), Project of Excellent talent "RiXin" of Beijing University of Technology.

\section{References}

[1] Y.F. Zhu, Student physical health affecting factors and scholastic physical education, J. Phys. Edu. 13 (2006) 141-144. In Chinese.

[2] C.M. Zhu, Y.M. Zhang, The advancement and developing trend of physical fitness study in China, China. Sport Sci. 28 (2008) 25-32.

[3] G.H. Yang, G.Q. Yang, Research on the effective evaluation system of the University's Sunshine Sports Movement, China. Adul. Edu. 24 (2011) 131-133.

[4] S.Z. Xiao, X.L. Fang, Z.F. Zhan, X.B. Sun, Z.F. Xiao, Research on a new method and index for evaluating cardiac function of the athletes, China. Med. Dev. Infor. 4 (2002) 34-36.

[5] X.M. Guo, Y. Yang, S.Z. Xiao, M.L. Xie, W.Z. Wu, Student physical health affecting factors and scholastic physical education, Chin. J. Appl. Physiol. 26 (2010) 380-383.

[6] S.L. Zhang, G.Z. Zou, J. Zhang, J. Wang, C.L. Yu, C. Wu, Z.P. Wang, J. Zhang, The design of the evaluation system for the sport technique and the effect to the institute of physical education, Sports. Sci. 21 (2000) 51-54.

[7] R. Li, C.M. Jiang, R. Rui, M. Wang, Post-exercise recovery heart rate reflecting heart fitness - limitation of fitness index in evaluation of heart fitness associated with increasing age, China. Sport. Sci. 32 (2012) 81-84.

[8] Q.M. Cai, Study on methods of evaluating physical fatigue according to dynamic heart rate, Chinese. Ergon. 5 (1999) 27-30.

[9] B. Liao, H. Gao, N. Chen, Methods for classifying intensity of physical labor based on relative heart rate, J. Saf. Sci. Tech. 9 (2013) 166-170. 